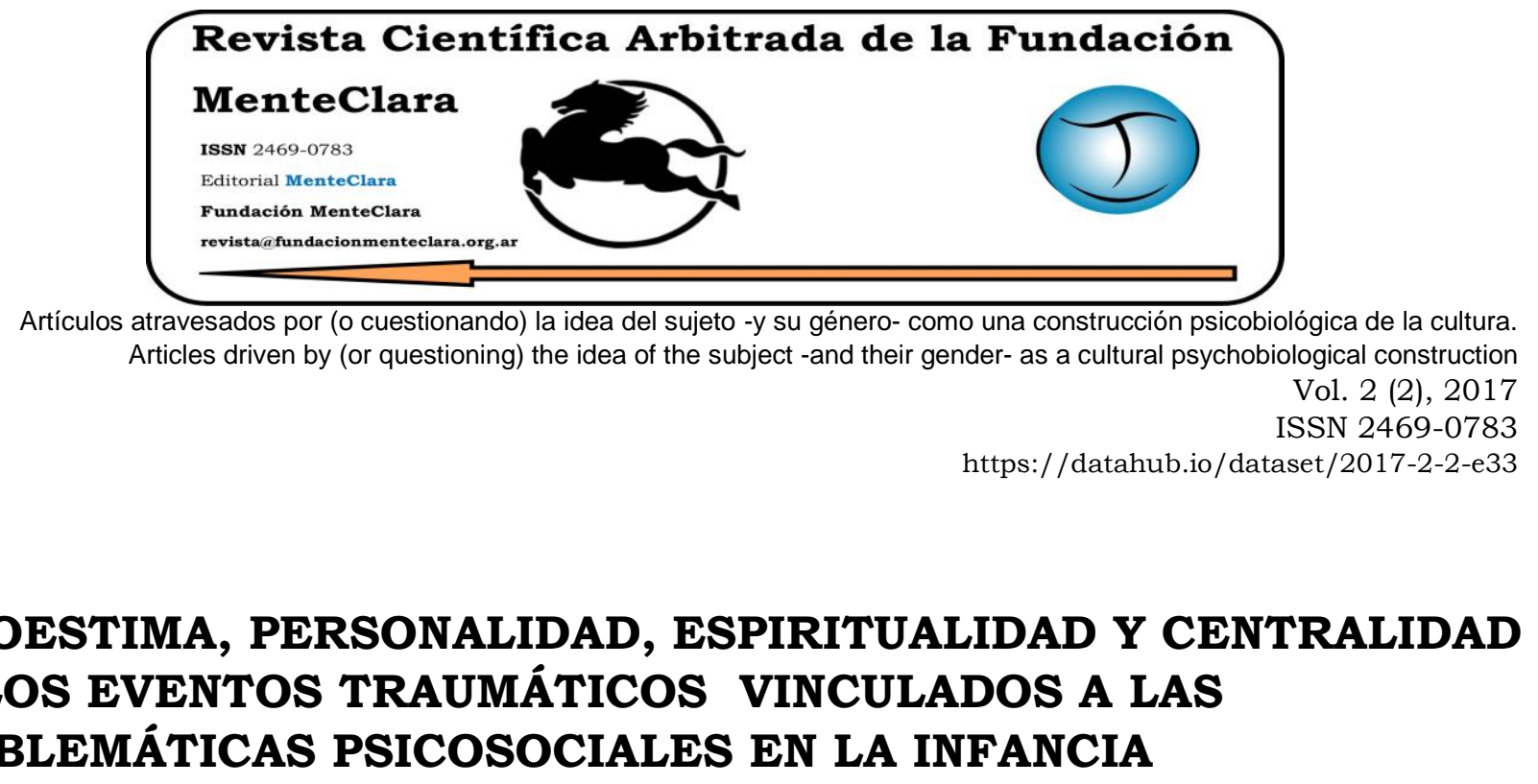

\title{
AUTOESTIMA, PERSONALIDAD, ESPIRITUALIDAD Y CENTRALIDAD DE LOS EVENTOS TRAUMÁTICOS VINCULADOS A LAS PROBLEMÁTICAS PSICOSOCIALES EN LA INFANCIA
}

\author{
SELF-ESTEEM, PERSONALITY, SPIRITUALITY AND CENTRALITY OF TRAUMATIC \\ EVENTS LINKED TO PSYCHOSOCIAL PROBLEMS IN CHILDREN \\ Gisela Matrángolo ${ }^{1}$, Cecilia Yaccarini², Gabriel Paz ${ }^{3}$
}

Cómo citar este artículo / Citation: Matrángolo G., Yaccarini C., Paz G. (2017). "Autoestima, personalidad, espiritualidad y centralidad de los eventos traumáticos vinculados a las problemáticas psicosociales en la infancia". Revista Científica Arbitrada de la Fundación MenteClara, 2(2), 217-237. DOI: 10.32351/rca.v2.2.35

Copyright: (C) 2017 RCAFMC. Este artículo de acceso abierto es distribuido bajo los términos de la licencia Creative Commons Attribution-Non Commercial (by-cn) Spain 3.0. Recibido: 01/10/2017. Aceptado: 25/10/2017 Publicación online: $30 / 10 / 2017$

Conflicto de intereses: Ninguno que declarar.

Este trabajo fue patrocinado en en forma conjunta por la Universidad Maimónides y la Universidad de Buenos Aires.

\section{Resumen}

La infancia se configura como un periodo de alta vulnerabilidad frente a distintas problemáticas psicosociales como son (1) La insatisfacción con la imagen corporal (2) El Bullying y Ciberbullying (3) El Maltrato hacia los niños (4) El rendimiento académico (6) Las adicciones (7) El trabajo en los niños (Simkin, Azzollini, \& Voloschin, 2014). Estas problemáticas, según distintos estudios, se encuentran estrechamente vinculadas a la formación de la Autoestima. Al tiempo que se observan relaciones entre estas, la Centralidad de los Eventos Traumáticos y el

\footnotetext{
${ }^{1}$ Universidad Maimónides / CONICET, Argentina. matrangolo.gisela@maimonides.edu

2 Universidad Maimónides, Argentina.

${ }^{3}$ Universidad de Buenos Aires, Argentina.
} 
surgimiento de síntomas de Trastorno por estrés postraumático y depresión. Se realizó una revisión bibliográfica en distintas bases de datos como Scielo, Latindex, Redalyc, PubMed, entre otras, con el objetivo de integrar los resultados de las investigaciones que asocian tales problemáticas a la autoestima en la niñez, atendiendo especialmente a la centralidad de los eventos como mediador del surgimiento de síntomas de estrés postraumático en la adultez, en el marco del Modelo y la Teoría de los Cinco Factores de la personalidad y la espiritualidad considerada como un sexto factor de la personalidad. Los resultados permiten concluir que el FFM y el FFT, tomando a la espiritualidad como un sexto factor constituyen una propuesta teórica robusta que facilitaría la posibilidad de comprender las relaciones entre los constructos mencionados.

\section{Abstract}

Childhood is configured as a period of high vulnerability to various psychosocial problems such as (1) Dissatisfaction with body image (2) Bullying and Cyberbullying (3) Child abuse (4) Academic performance (6) Addictions (7) Work in children (Simkin, Azzollini, \& Voloschin, 2014). These problems, according to different studies, are inextricably linked to the formation of Self-esteem. At the same time, there are relationships between these, the Centrality of Traumatic Events and the emergence of symptoms of Post-Traumatic Stress Disorder and depression. A literature review was carried out in different databases such as Scielo, Latindex, Redalyc, PubMed, among others, with the aim of integrating the results of research that associate such problems with self-esteem in childhood, paying particular attention to the centrality of events as a mediator of the emergence of symptoms of post-traumatic stress in adulthood, within the framework of the Model and Theory of the Five Factors of personality and spirituality considered as a sixth factor of personality. The results allow us to conclude that the FFM and the FFT, taking spirituality as a sixth factor constitute a robust theoretical proposal that would facilitate the possibility of understanding the relationships between the aforementioned constructs.

\section{Palabras Claves/ Keywords}

PROBLEMÁTICAS PSICOSOCIALES; AUTOESTIMA; FFM; ESTRÉS POSTRAUMÁTICO; CENTRALIDAD DE LOS EVENTOS; PSYCHOSOCIAL PROBLEMS; SELF-ESTEEM; POSTRAUMATIC STRESS; CENTRALITY OF EVENTS 


\section{Introducción.}

Durante la infancia se han identificado una serie de problemáticas psicosociales que podrian impactar negativamente en el desarrollo de la autoestima sujeto. A continuación se indicarán aquellas que son de particular interés para este trabajo: (1) el aspecto fisico en la infancia (2) el desempeño académico (3) El Bullying y Ciberbullying (4) El Maltrato Infantil (5) El trabajo Infantil (6) las adicciones en la infancia (Simkin, Azzollini, \& Voloschin, 2014). Es posible que esta relación siente sus bases en creencias culturales que invitan a suponer que el valor de una persona se mide en relación al éxito que esta ha obtenido en relación a objetivos socialmente presentados como valiosos, partiendo de la idea de que cada persona obtiene lo que se merece - meritocracia-, de acuerdo al esfuerzo individual realizado que se disponga a realizar, así según afirman ciertos autores, la autoestima es incluso protegida por la idea de meritocracia que señala que aquellos sectores más desfavorecidos son responsables de su propia desdicha(Gerosa, 2016; Mccoy, Wellman, \& Cosley, 2014). En los casos que estas experiencias, potencialmente traumáticas, se configuren como puntos de inflexión en la vida del sujeto, transformándose en un componente central de la identidad personal, además de impactar negativamente en la autoestima, podría presentarse, sintomatología de Trastorno de Estrés Postraumático (Berntsen \& Rubin, 2006; Ogle, Rubin, \& Siegler, 2014).Guiados por estudios anteriores, se propone integrar los constructos mencionados en el marco del Modelo y Teoría de los Cinco Factores de la Personalidad a fin de comprender en mayor medida las interacciones entre estos (H. Simkin \& Azzollini, 2015). Dado que es escaso el desarrollo, en el contexto local, de investigaciones que vinculen a las problemáticas psicosociales en la infancia, la autoestima, la centralidad de los eventos traumáticos y el surgimiento de síntomas de Trastorno de Estrés Postraumático, el objetivo del presente 
trabajo consiste en revisar los antecedentes en el área, integrando los resultados de las investigaciones en el marco del Modelo y la Teoría de los Cinco Factores de la Personalidad.

\section{Infancia y autoestima}

La Infancia como categoría social, surge a partir de un proceso socio histórico, con el objeto de identificar un determinado grupo social al que se le confieren características específicas (Colángelo, 2003). Así, se considera a la infancia como una realidad construida socialmente, permeable a variaciones culturales e históricas, marcadas por un conjunto de pautas, normas de conducta y valores que se vinculan al modo de ser niño en un momento concreto, en sintesis, los niños, al igual que los adultos están sujetos a las vicisitudes de los cambios sociales (Gaitán, 2006; Pavez Soto, 2012). Se destaca que la primera infancia es considerada un período clave en la constitución del ser humano debido a que en este se producen importantes procesos de maduración y desarrollo a nivel físico, psicológico, emocional, intelectual y social que sentaran las bases a partir de las cuales el niño se diferenciará del mundo y comenzará a autoevaluarse en función a los logros percibidos y de comparaciones con pares (Camargo-Ramos, \& Pinzon-Villate, 2012). Durante el proceso de socialización, los distintos agentes que se relacionan con el niño, educadores, padres, pares, medios de comunicación, referentes de instituciones religiosas y deportivas transmiten ideales, valores y creencias al niño que funcionan como un modelo a partir del cual este se autoevalúa (Pierce \& Wardle, 1997; Rosemberg,1965). Cabe considerar que la definición de autoestima más influyente hasta hoy fue acuñada por James en el año 1890, este autor hizo especial hincapié en el carácter individual del concepto de 
autoestima considerándolo como la medida en que las personas se evalúan a sí mismas de acuerdo al éxito o fracaso percibido en alcanzar sus objetivos, esta evaluación es subjetiva, central y no se basa en conductas especificas (Caminos, \& Amichetti Quentrequeo, 2015; DiLillo et al., 2010; Leary \& Baumeister, 2000; Sánchez \& Cuenya, 2011; Sowislo \& Orth, 2013). Se considera, de acuerdo a la bibliografia relevada que la autoestima comienza a configurarse en etapas tempranas de la niñez a partir de las autobservaciones y autoevaluaciones de sus capacidades, comparando su rendimiento actual con desempeños previos propios, y con el desempeño de los otros. Así, la formación del autoconcepto y de la autoestima surge a parir de la interacción con los otros significativos (Serrano Muñoz, Mérida Serrano, \& Tabernero Urbieta, 2015). En cuanto al desarrollo de la autoestima, a lo largo de la infancia se observa que si bien, en general, en la infancia temprana los sujetos presentan una autoestima alta, en momentos posteriores, especialmente, en cercanías de la pubertad los niveles de autoestima tienden a bajar (Silverthorn et al., 2017).

\section{Problemáticas Psicosociales en la Infancia}

Actualmente se han identificado diferentes problemáticas psicosociales que impactan en la formación de la autoestima durante la infancia:

(1) El aspecto físico en la Infancia:

El desarrollo de la autoestima durante la infancia se encuentra estrechamente vinculado a la valoración del aspecto físico, por lo cual presentar características fisicas socialmente valoradas se constituiría como una fuente de autoestima, mientras que, por el contrario, presentar características como el sobrepeso u obesidad, dada su estigmatización 
social socavaria la autoestima (Molina \& Raimundi, 2011; Pierce \& Wardle, 1997). A este respecto se observa que, en gran medida son los medios de comunicación los que tienden a presentar una serie de ideales de belleza que se constituyen como parámetros a partir de los cuales compararse y evaluarse. Diferentes estudios han observado que los medios de comunicación desempeñan un papel central en la formación de ideales culturales de belleza, que constituyen los parámetros en base a los cuales niños se autoevalúan (Montoya, 2015; Sánchez; López; Fauquet, 2014). Estos ideales son reforzados, por un lado, por los padres quienes suelen transmitir a sus hijas preocupaciones en relación a la estética hegemónica, imponiendo la necesidad de perder peso, cuestión que contribuye a que estas desarrollen una preocupación excesiva por su apariencia (Facchini, 2006; Gimenez, Correché,\& Rivarola, 2013). Por otro lado, se observa que el grupo de pares influye en relación a la percepción negativa del niño, y especialmente de la niña acerca de su propia imagen y las conductas dietarias de estas últimas (Facchini, 2006; Rosés Gómez, Raich, \& Mora Giral, 2015).

(2) Desempeño académico en la Infancia:

Según Duru y Balkis (2014) Posiblemente debido a la importancia que presenta a nivel social la escolaridad durante la infancia, se observa que la autoestima se vincula en forma estrecha, dinámica y recíproca al rendimiento académico, de manera que un desempeño académico bajo generaria una disminución de la autoestima, al tiempo que una autoestima baja, sería una de las causas de la disminución de la efectividad en el desempeño académico. Sin embargo, otros autores indican que una autoestima alta no repercute en sí en el rendimiento académico, sino que un buen desempeño académico es uno de los 
factores que influyen en el incremento de la autoestima (Baumeister, Campbell, Krueger, \& Vohs, 2003; Morente, Guiu, Castells, \& Escoda, 2017; Zuffianò et al., 2013). En nuestra cultura, el rendimiento académico suele ser altamente valorado dentro del contexto familiar (Farris, Burke Lefever, Borkowski, \& Whitman, 2013) y reforzado en el contexto escolar, sin embargo, si el niño proviene de una cultura en la que no se juzga importante la escuela, su autoestima quizá no se encuentre relacionada con el logro académico, sino que dependa del grado de competencia alcanzado en aquellas áreas valoradas por su entorno.

(3) Bullying y Ciberbullying en la infancia:

El bullying puede definirse como acciones negativas fisicas o verbales que se llevan a cabo en la escuela, en el aula o en cualquier otro sector, que tienen intenciones hostiles, se repiten en el tiempo, e implican una diferencia de poder entre el agresor y la víctima. El acoso escolar puede darse en relación a cuestiones académicas (rendimiento, disciplina, orden) o aspectos físicos (sobrepeso, tono de voz, rasgos del rostro, color de piel, cicatrices, postura corporal, etc.) cualquiera de estas expresiones del escolar son motivo para elegirlo centro de acoso ocasional o permanente (Scott \& Dale, 2016). A partir de los tres años pueden emerger conductas consideradas como bullyng (Vlachou, Andreou, Botsoglou, \& Didaskalou, 2011) al hacer gestos de burla o reprobación dirigidos intencionalmente a otros niños, como por ejemplo: amenazar, romper objetos, empujar o excluir a otros para defender sus posesiones, el territorio y las amistades o demostrar la superioridad y dominación sobre sus pares. En relación al género, los varones tienden a percibir más frecuentemente la conducta de sus compañeros como burlas que las 
niñas. Podría pensarse que los niños son más proclives a manifestar este tipo de agresión verbal, o que son más sensibles a este tipo de manifestaciones que las niñas (Gorostegui, \&Dörr, 2005).

De acuerdo con Espelage y De La Ruev (2012), el comportamiento de bullying es modelado por diversas cuestiones interrelacionadas, como las características individuales, familiares, el grupo de pares y el entorno escolar. En el marco de la familia, los niños que experimentan u observan la violencia familiar, castigo físico, o el uso de la agresión verbal o fisica para controlar a los demás tienden a presentar un comportamiento intergrupal más agresivo. Un estilo de crianza autoritario incrementa la posibilidad de ser victima o victimario de bullying durante la infancia (Simkin, Azzollini, \& Voloschin, 2014).

Las nuevas tecnologias han revolucionado, desde hace varios años, la forma de comunicarse, construirse y comprenderse dentro del marco de las relaciones sociales. La masificación de los medios de comunicación, en vertiginoso crecimiento en nuestros dias ha dado lugar tanto a grandes progresos como a nuevas situaciones problemáticas. E1 Cyberbullying puede ser entendido como conductas de acoso que infligen daño de forma deliberada, intencional y repetida a un sujeto a través del uso de dispositivos electrónicos como pc, tablets o teléfonos celulares (Patchin \& Hinduja, 2010)

El Cyberbullying es considerado una de las formas de acoso de más alcance debido a que la información se difunde con facilidad y rapidez, quedando además, disponible a través del tiempo, incluso al alcance de ser visto por personas ajenas a la situación de hostigamiento inicial lo que lo convierte en una forma de asedio particularmente perdurable y corrosiva, que al igual que el bullying se asocia negativamente con la 
autoestima de la víctima (Brewer \& Kerlaske, 2015) (Patchin \& Hinduja, 2010).

(4) Autoestima y maltrato en la infancia

Las conductas de maltrato infantil suelen ocurrir en el marco de las prácticas de crianza en la interacción entre padres e hijos, especialmente en la implementación de pautas disciplinares (Rodriguez, 2016). En este sentido, distintos estudios arrojan que frecuentemente, aquellos adultos que han sufrido distintas formas de maltrato en su infancia tienden a reproducirlas sobre sus hijos, de modo que se refuerza la idea de que las pautas de crianza son, en gran medida, aprendidas a partir de la propia experiencia del cuidador (Moreno, 2014; Rodriguez, 2016; RomeroMartínez, Figueiredo, \& Moya-Albiol, 2014). En necesario remarcar que, en relación a los tipos de maltrato infantil, se observa que la mayoría de los estudios se abocan a cuestiones relacionadas al abuso sexual y el maltrato fisico, en detrimento del interés por el maltrato emocional y los dos tipos de negligencia, aunque son conocidas la tendencia a la cronicidad y las consecuencias de estos tipos de maltrato (Matrángolo, 2016; Spinhoven, Penninx, Hickendorff, Hemert, \& Bernstein, 2014). De acuerdo a un estudio realizado en Argentina, se observa también que el maltrato infantil es más prevalente entre los 0 y los 5 años, remarcándose la vulnerabilidad de este grupo etario (Brignotti, 2008). A partir de otro estudio, realizado también en el contexto local, se observa además, que el tipo de maltrato sufrido se relaciona al género de la víctima. De esta manera se observa que son las niñas quienes presentan mayor porcentaje de abuso sexual $26,6 \%$ frente a $10 \%$ en varones, mientras que estos últimos fueron, en mayor medida, víctimas de negligencia y maltrato físico $24,7 \%$ y $47,3 \%$ respectivamente, frente a un $16,9 \%$ y $41,3 \%$ es 
menester aclarar que en el caso de este estudio la muestra, compuesta por 5341 niños y adolescentes, que han sufrido vulneración de sus derechos, se extrajo de la base REUNA -Registro Estadístico Unificado de Niñez y Adolescencia- a partir intervenciones realizadas por los 22 Servicios Zonales de Promoción y Protección de Derechos del Niño (SZPPD) de la Provincia de Buenos Aires (Sánchez \& Cuenya, 2011).Numerosos estudios vinculan el maltrato infantil con la autoestima, indicando, en su mayoria que el maltrato infantil disminuye la autoestima (Edwards, Holden, Felitti, \& Anda, 2003; Greger, Myhre, Klöckner, \& Jozefiak, 2017; Stein, Leslie, \& Nyamathi, 2002).

(6) Autoestima y Trabajo en la Infancia

Todo niño tiene derecho a la educación y a estar protegido contra cualquier tipo de trabajo que ponga en riesgos o limite su educación y desarrollo integral (Convención de los Derechos del niño, naciones unidas, 1989). La Organización Internacional del Trabajo -OIT- considera al trabajo infantil como toda actividad económica llevada a cabo por personas menores de 15 años de edad, sin incluir las tareas del hogar, excepto que puedan ser considerada una actividad económica (Padrón Innamorato,\& Gonzalez Contró, 2012). A partir de esta consideración de los derechos fundamentales se propone la pregunta: ¿Qué factores llevan a que los niños, prematuramente, se integren al mundo laboral? Los motivos por los que los niños y niñas trabajan son diversos comprendiendo factores estructurales, culturales, sociales y económicos (Sandoval Avila, 2007). Paz y Piselli (2011), en Argentina, observan que a pesar de la relación que a nivel descriptivo puede hallarse, ni la pobreza monetaria ni las privaciones materiales son determinantes cruciales y de 
peso en la decisión de los hogares de hacer trabajar a niños y adolescentes.

La mayoria de los estudios que tratan sobre la relación entre trabajo infantil y calidad de vida, parten de la premisa de que la escuela es el "único lugar natural de la infancia" y cualquier forma de trabajo es una actividad destinada a negarle al niño la posibilidad de una niñez saludable (Morales Topanto, 2014). Distintos estudios indican que el trabajo infantil, por lo menos en población occidental tiende a disminuir el autoestima de los niños (Santacruz, 2013).

(7) Autoestima y Adicciones en la Infancia:

Puede entenderse a las adicciones como la repetición continuada de un comportamiento, que a pesar de acarrear consecuencias adversas o un deterioro neurológico continúan llevandose a cabo (Angres \& Bettinardi-Angres, 2008; Catalina-Vicol, Stinga, Caba, Ioan, \& Astarastoae, 2008). El DSM-5 (APA, 2013) considera únicamente la adicción a sustancias y a juegos de azar, soslayando adicción a juegos electrónicos, a las redes sociales, a la comida, al uso de internet, etc.

Los resultados obtenidos en las últimas décadas en cuanto a la relación entre las adicciones y la autoestima han resultado inconsistente (Donnelly et al., 2008; Kavas, 2009), posiblemente debido a limitaciones conceptuales y metodológicas, problemas en la evaluación de los constructos y la mala interpretación de los datos estadísticos (Kounenou, 2010). Según Kaden y Litt, 2011 uno de los motivos que podría explicar la asociación negativa entre la autoestima y la adicción se enraíza en el impacto negativo que presenta la adicción en la autoeficacia. Sin embargo, la aceptación social se encuentra dividida: mientras que algunos miembros del entorno pueden mostrar preocupación, otros 
(generalmente pares) pueden a reforzar la conducta adictiva (Ventura, de Souza, Hayashida, \& Ferreira, 2015). Por este motivo, resulta necesario continuar realizando estudios que puedan discriminar cómo y de qué manera las diferentes adicciones se encuentran asociadas a la autoestima.

\section{La centralidad de los eventos traumáticos}

El Trastorno por Estrés Postraumático (TEPT) se compone por cuatro grupos de sintomas fundamentales, que surgen a partir de una situación o hecho traumático, que el sujeto ha experimentado, presenciado o le han contado y al que ha reaccionado con indefensión o terror intenso (Boh, Mun, Londo, \& Vinaccia, 2016; Crespo \& Gomez, 2012; Echeburúa, Amor, Muñoz, Sarasua, \& Zubizarreta, 2017; Echeburúa, de Corral, Amor, Zubizarreta, \& Sarasua, 1997). Los cuatro grupos de sintomas fundamentales en el TEPT son (1) Reexperimentación, (e.g. Sueños recurrentes en relación a la situación traumática), (2) Conductas evitativas ( e.g. Evitar comunicarse con personas relacionadas a este), (3) Alteraciones cognitivas negativas (e.g. Creencias o expectativas negativas acerca de uno mismo o los demás) (4) Aumento de la Activación y reactividad psicofisiológica (e.g. Estado de alerta permanente) (Echeburúa et al., 2017, 1997). Se ha observado que, las relaciones entre las experiencias traumáticas y la prevalencia de síntomas de estrés postraumático se encuentran moduladas por la forma en que los individuos integran los eventos potencialmente traumáticos a su propio self, entendido este como la concepción que tenemos sobre nosotros, como individuos, o como pertenecientes a un grupo (Páramo, 2008). En relación a esta cuestión, existen dos posturas fundamentales: por un lado, algunos autores afirman que la dificultad en integrar el evento 
vivido por el sujeto como traumático, debido a su carga emocional negativa, en la memoria autobiográfica, genera que los recuerdos del incidente permanezcan fragmentados y no integrados al self del sujeto, por lo que los posibles posteriores síntomas de estrés post traumático se vincularian con esta dificultad en la integración (Dalgleish, 2004; Dalgleish, Rolfe, Golden, Dunn, \& Barnard, 2008; Kleim, Wallott, \& Ehlers, 2008). Por otro lado, Berntsen y Rubin (2007) plantean que cuando un evento traumático se configura como un punto de inflexión en la vida de la persona, es decir cuando el evento se configura como central en su vida, la posibilidad de desarrollar síntomas de estrés postraumático se incrementa. Entonces, el evento traumático pasa a formar parte de la identidad del sujeto, conformando una red compleja con otros recuerdos que forman parte de su self e influyendo en el modo en el que procesa la información del mundo circundante (Berntsen \& Rubin, 2007; Berntsen, Willert, \& Rubin, 2003).

Integración de las Problemáticas Psicosociales, la Autoestima y la centralidad de los eventos en el Marco del Modelo y la Teoria de los Cinco Factores.

En el presente trabajo, en base a los antecedentes se plantea la necesidad de analizar las problemáticas psicosociales relacionada a la centralidad de los eventos y la autoestima en el marco del Modelo y la Teoría de los Cinco Factores De la Personalidad, considerando la inclusión de la espiritualidad como integrada a este modelo, y por lo tanto, apreciado como un sexto factor. A partir de distintos trabajos se ha intentado integrar el vínculo entre los rasgos de la personalidad, la religiosidad, la espiritualidad y la autoestima en el marco del Modelo y la Teoría de los Cinco Factores (Gerosa, 2016; Simkin \& Azzollini, 2015) .Sin 
duda, el modelo de los Cinco Factores es uno de los más empleados en para el estudio de la personalidad (Simkin \& Azzollini, 2015). Según las bases de este Modelo, los rasgos de la personalidad pueden organizarse en cinco dimensiones básicas siendo estas Neuroticismo, Extraversión, Apertura a la experiencia, Amabilidad y Responsabilidad (Costa \& McCrae, 2005, 2012; Costa, Patriciu, \& McCrae, 2005; Gerosa, 2016; McCrae, 2009). Desarrollos actuales sugieren incluir a la espiritualidad como un sexto factor de la personalidad (Piedmont, 1999b; Piedmont \& Wilkins, 2013; Simkin, 2017). Estos factores impactarian en la autoestima entendida como característica adaptativa, a la vez que ésta podria resultar afectada por las influencias externas (Simkin \& Azzollini, 2015).De acuerdo a los antecedentes, los agentes de socialización transmiten un conjunto de valores de , un sistema de creencias e ideales que proporcionan las bases para autoevaluarse, así los medios de comunicación, los docentes, los referentes deportivos y religiosos, los padres y los gupos de pares pueden contribuir a reforzar un determinado ideal cultural (Mccoy et al., 2014; Sánchez; López,; Fauquet, 2014; Silverthorn et al., 2017) ya sea un ideal cultural de belleza a partir del cual compararse. Se identificaron distintas problemáticas sociales que surgen en el marco del proceso de socialización e impactan en la autoestima, al tiempo que pueden constituirse como eventos traumáticos si terminan por configurarse como centrales en la identidad del sujeto. La satisfacción con el propio aspecto físico (Mccoy et al., 2014; Rosés Gómez et al., 2015; Silverthorn et al., 2017), el rendimiento académico (Zuffianò et al., 2013), el bullying o ciberbullying (Scott \& Dale, 2016; Vlachou et al., 2011; Voloschin, Becerra, \& Simkin, 2016), el maltrato infantil (Edwards et al., 2003; Greger et al., 2017), el trabajo infantil (Ávila, 2007), o las adicciones en la infancia (Kounenou, 2010) se configuran como problemáticas psicosociales básicas. Por otra parte, es 
necesario destacar que, según la revisión realizada los rasgos de la personalidad se vincularian a la posibilidad de alcanzar o no los ideales culturales que se proponen socialmente. En primer lugar, la responsabilidad se expresa en la capacidad de planificar y sistematizar esfuerzos en pos de alcanzar un objetivo; En segundo lugar, la extroversión se caracteriza por reflejarse en una alta eficacia social: En tercer lugar las personas con mayor amabilidad, suelen ser gentiles y cooperativos, estos tres factores presentan vínculos positivos con la autoestima (McCrae \& Löckenhoff, 2010). Por su parte, la Apertura y el Neuroticismo pueden asociarse negativamente a la autoestima (Piedmont, Sherman, \& Sherman, 2012). En relación a la espiritualidad se observan vínculos positivos con la autoestima, esto podría deberse a que la autotrascendencia espiritual tiende promover el desapego, permitiendo desalienarse de los ideales establecidos y permitiendo aceptar la vida y los otros tal cual son (Coholic, 2005; Simkin \& Azzollini, 2015; Simkin \& Etchevers, 2014).

\section{Conclusión}

De acuerdo con la revisión realizada, diferentes problemáticas psicosociales tales como el acoso escolar, el maltrato infantil y la preocupación por el aspecto físico afectan la autoestima en los niños (Ajmal, Shamim, \& Saleh, 2016; Mccoy et al., 2014; Ozbas, Gurhan, \& Kocak, 2013; Scott \& Dale, 2016; Vlachou et al., 2011). Estos acontecimientos, por su carga emocional negativa pueden, además, de disminuir la autoestima, potencialmente configurarse como traumáticos. A este respecto, Berntsen y Rubin (2007) plantean que cuando un evento traumático se configura como un punto de inflexión en la vida de la persona, es decir cuando el evento se configura como central en su vida, 
la posibilidad de desarrollar sintomas de estrés postraumático se incrementa. Entonces, el evento traumático pasa a formar parte de la identidad del sujeto, conformando una intrincada red con otros recuerdos que forman parte de su self e influyendo en el modo en el que procesa la información del mundo circundante e incrementando la posibilidad del surgimiento de sintomatología de Estrés Postraumático (Berntsen \& Rubin, 2007; Berntsen et al., 2003). El FFM y el FFT se presentan como un marco integrador que permite comprender cómo las influencias externas y la cultura interaccionan, dando lugar a características adaptativas como el autoconcepto y la autoestima. La inclusión de la espiritualidad, como un sexto factor de la personalidad, sin duda, robustece el modelo ya que permite considerar características como el desapego de las definiciones externas del self y de los parámetros de éxito y fracaso internalizados en el proceso de socialización que promovería cierto desinterés por perseguir el éxito y menores sintomas de estrés postraumático. Será tarea de futuras investigaciones contribuir a comprender su lugar en el marco del Modelo y la Teoría de los Cinco Factores. 


\section{Referencias}

Ajmal, M. M., Shamim, M., \& Saleh, M. (2016). Journal of Behavioural Sciences, Vol. 26, No. 2, 2016, 26 (2).

Angres, D. H., \& Bettinardi-Angres, K. (2008). The Disease of Addiction: Origins, Treatment, and Recovery. Disease-a-Month, 54(10), 696-721.

https://doi.org/10.1016/j.disamonth.2008.07.002

Ávila, A. S. (2007). Trabajo infantil e inasistencia escolar. Revista Brasileira de Educação, 12(34), 68-80. https://doi.org/10.1590/S1413-24782007000100006

Baumeister, R. F., Campbell, J. D., Krueger, J. I., \& Vohs, K. D. (2003). Does high self esteem cause better performance, interpersonal success, happines or healthier lifestyle? Psychological Science in the Public Interest, 4(1), 1-44.

Ben-David, V., \& Jonson-Reid, M. (2017). Resilience among adult survivors of childhood neglect: A missing piece in the resilience literature. Children and Youth Services Review, 78(December 2016), 93-103. https://doi.org/10.1016/j.childyouth.2017.05.014

Berntsen, D., \& Rubin, D. C. (2006). The centrality of event scale: A measure of integrating a trauma into one's identity and its relation to post-traumatic stress disorder symptoms. Behaviour Research and Therapy, 44(2), 219-231. https://doi.org/10.1016/j.brat.2005.01.009

Berntsen, D., \& Rubin, D. C. (2007). When a Trauma Becomes a Key to Identity : Enhanced Integration of Trauma Memories Predicts Posttraumatic Stress Disorder Symptoms. Applied Cognitive Psychology, 21, 417-431. https://doi.org/10.1002/acp

Berntsen, D., Willert, M., \& Rubin, D. C. (2003). Splintered memories or vivid landmarks? Qualities and organization of traumatic memories with and without PTSD. Applied Cognitive Psychology, 17(6), 675-693. https://doi.org/10.1002/acp.894

Boh, D., Mun, F. R., Londo, C., \& Vinaccia, S. (2016). El sistema inmunológico en personas con trastorno de estrés postraumático: una revisión, 26(57), 245-252.

Brignotti. (2008). Elevamiento epidemiologico en la poblacion escolari - zada de la ciudad autonoma de buenos aires. Ciencias Psicológicas, 2(2), 131-141.

Camargo-Ramos, C., Pinzon-Villate, G. (2012). La promoción de la salud en la primera infancia: evolución del concepto y su aplicación en el contexto internacional y nacional. Rev Fac Med.Suplemento, 60(11), 62-74.

Caminos, M., \& Amichetti Quentrequeo, A. (2015). Eteronormatividad, autoestima y bullying homofóbico en Argentina. Psocial, 1(2), 17-32.

Catalina-Vicol, M., Stinga, O. C., Caba, L., Ioan, B., \& Astarastoae, V. (2008). Drug Adiction - it is just a Medical Probelem? Revista Romana De Bioetica, 6 (4), 98-110.

Coholic, D. (2005). The Helpfulness of Spiritually Influenced Group Work in Developing Self-Awareness and Self-Esteem: A Preliminary Investigation. The Scientific World JOURNAL, 5, 789-802. https://doi.org/10.1100/tsw.2005.99

Colángelo, M. A. (2003). La mirada antropológica sobre la infancia. Reflexiones y perspectivas de abordaje. Infancias Y Juventudes. Pedagogia Y Formacion, 1-8. Retrieved from http://www.me.gov.ar/curriform/publica/oei.../ponencia_colangelo.pdf

Costa, P. T., \& McCrae, R. R. (2005). A Five-Factor Theory Perspective on the Rorschach. Rorschachiana, 27(1), 80-100. https://doi.org/10.1027/1192-5604.27.1.80

Costa, P. T., \& McCrae, R. R. (2012). The Five-Factor Model, Five-Factor Theory, and Interpersonal Psychology. In L. M. Horowitz \& S. Strack (Eds.), Handbook of 
Interpersonal Psychology: Theory, Research, Assessment, and Therapeutic Interventions (pp. 91-105). New Jersey: John Wiley \& Sons.

https://doi.org/10.1002/9781118001868.ch6

Costa, P. T., Patriciu, N. S., \& McCrae, R. R. (2005). Lessons from Longitudinal Studies for New Approaches to the DSM-V: The FFM and FFT. Journal of Personality Disorders, 19(5), 533-539. https://doi.org/10.1521/pedi.2005.19.5.533

Crespo, M., \& Gomez, M. M. (2012). La Evaluación del Estrés Postraumático: Presentación de la Escala de Evaluación Global de Estrés Postraumático (EGEP). Clínica Y Salud, 23(1), 25-41. https://doi.org/10.5093/cl2012a4

Dalgleish, T. (2004). Cognitive Approaches to Posttraumatic Stress Disorder: The Evolution of Multirepresentational Theorizing. Psychological Bulletin, 130(2), 228-260. https:/ / doi.org/10.1037/0033-2909.130.2.228

Dalgleish, T., Rolfe, J., Golden, A.-M., Dunn, B. D., \& Barnard, P. J. (2008). Reduced autobiographical memory specificity and posttraumatic stress: Exploring the contributions of impaired executive control and affect regulation. Journal of Abnormal Psychology, 117(1), 236-241. https://doi.org/10.1037/0021-843X.117.1.236

DiLillo, D., Hayes-Skelton, S. A., Fortier, M. A., Perry, A. R., Evans, S. E., Messman Moore, T. L., ... Fauchier, A. (2010). Development and initial psychometric properties of the Computer Assisted Maltreatment Inventory (CAMI): A comprehensive self-report measure of child maltreatment history. Child Abuse and Neglect, 34(5), 305-317. https://doi.org/10.1016/j.chiabu.2009.09.015

Donnelly, J., Young, M., Pearson, R., Penhollow, T. M., \& Hernandez, A. (2008). Area Specific Self-Esteem, Values, and Adolescent Substance Use. Journal of Drug Education, 38(4), 389-403. https://doi.org/10.2190/DE.38.4.f

Echeburúa, E., Amor, P. J., Muñoz, J. M., Sarasua, B., \& Zubizarreta, I. (2017). Escala de Gravedad de Síntomas del Trastorno de Estrés Postraumático según el DSM-5: versión forense (EGS-F). Anuario de Psicologia Juridica, 27(1), 67-77. https://doi.org/10.1016/j.apj.2017.02.005

Echeburúa, E., de Corral, P., Amor, P. J., Zubizarreta, I., \& Sarasua, B. (1997). Escala de gravedad de sintomas del trastorno de estrés postraumático: propiedades psicométricas. Análisis Y Modificación de Conducta. Retrieved from http://zutitu.com/FitxersWeb/20/ARTICULO10.pdf

Edwards, V. J., Holden, G. W., Felitti, V. J., \& Anda, R. F. (2003). Relationship between multiple forms of childhood maltreatment and adult mental health in community respondents: Results from the adverse childhood experiences study. American Journal of Psychiatry, 160(8), 1453-1460. https://doi.org/10.1176/appi.ajp.160.8.1453

Facchini, D. M. (2006). La preocupación por el peso y la figura corporal en las niñas y adolescentes actuales : ¿de dónde proviene?, 104(4), 345-350.

Farris, J., Burke Lefever, J. E., Borkowski, J. G., \& Whitman, T. L. (2013). Two Are Better Than One: The Joint Influence of Maternal Preparedness for Parenting and Children's Self-Esteem on Academic Achievement and Adjustment. Early Education and Development, 24(3), 346-365. https://doi.org/10.1080/10409289.2012.658551

Gaitán, L. (2006). La nueva sociología de la infancia. Aportaciones de una mirada distinta. Politica Y Sociedad, 43(1), 9-26. https://doi.org/-

Gerosa, F. (2016). PERSONALIDAD, AUTOESTIMA Y DESEMPEÑO ACADÉMICO. UNA APROXIMACIÓN COMPLEMENTARIA A PARTIR DE LA TEORÍA DE LOS CINCO FACTORES DE LA PERSONALIDAD. Psocial, 2, 46-53. 
Gimenez, P.;Correché, S.\& Rivarola, M. . (2013). Autoestima e Imagen Corporal . Estrategias de intervención psicológica para mejorar el bienestar psicológico en pre- adolescentes en una escuela de la ciudad de San Luis, Argentina.

Greger, H. K., Myhre, A. K., Klöckner, C. A., \& Jozefiak, T. (2017). Childhood maltreatment, psychopathology and well-being: The mediator role of global self-esteem, attachment difficulties and substance use. Child Abuse and Neglect, 7O(February), 122-133. https://doi.org/10.1016/j.chiabu.2017.06.012

John, D., Trauma, R. J. M., \& Link, C. (2017). Trauma Centrality and PTSD Symptom Severity in Adult Survivors of Childhood Sexual Abuse The Harvard community has made this article openly available. Please share how this access benefits you. Your story matters . Citation Accessed. https://doi.org/10.1002/jts.20656

Kleim, B., Wallott, F., \& Ehlers, A. (2008). Are Trauma Memories Disjointed from other Autobiographical Memories in Posttraumatic Stress Disorder? An Experimental Investigation. Behavioural and Cognitive Psychotherapy, 36(2), 221-34. https://doi.org/10.1017/S1352465807004080

Kounenou, K. (2010). Exploration of the relationship among drug use \& alcohol drinking, entertainment activities and self-esteem in Greek University students. Procedia - Social and Behavioral Sciences, 2(2), 1906-1910. https://doi.org/10.1016/j.sbspro.2010.03.1007

Leary, M. R., \& Baumeister, R. F. (2000). The nature and function of self-esteem: Sociometer theory, 32, 1-62. https://doi.org/10.1016/S0065-2601(00)80003-9

Lobbestael, J. (2009). Thombs BD, Bernstein DP, Lobbestael J , Arntz A . A validation study of the Dutch Childhood Trauma Questionnaire-Short ..., (August). https://doi.org/10.1016/j.chiabu.2009.03.001

Matrángolo, G. (2016). Maltrato Infantil: Herramientas para su Enaluación Retrospectiva. Una revisión Bibliográfica., \{PSOCIAL\}, 2 (2), 54-64.

Mccoy, S. K., Wellman, J. D., \& Cosley, B. (2014). NIH Public Access, 43(4), 307-318. https://doi.org/10.1002/ejsp.1959.Is

McCrae, R. R. (2009). The Physics and Chemistry of Personality. Theory \& Psychology, 19(5), 670-687. https://doi.org/10.1177/0959354309341928

McCrae, R. R., \& Löckenhoff, C. E. (2010). Self Regulation and the Five Factor Model of Personality Traits. In R. Hoyle (Ed.), Handbook of personality and self-regulation (pp. 145-168). West Sussex: Wiley-Blackwell.

Molina, M. F., \& Raimundi, M. J. (2011). Predictores de la autoestima global en niños de escuela primaria de la Ciudad de Buenos Aires. Diferencias en función del sexo y la edad. Revista Argentina de Ciencias Del Comportamiento, 3(3), 1-7.

Montoya, Y. et al. (2015). Influencia de los medios de comunicación y el riesgo de padecer trastornos de la conducta alimentaria en escolares mujeres en Lima , Perú, 113(6), 519525.

Moreno, J. (2014). Etiología del maltrato infantil: estilo educativo, prácticas de crianza y contexto social. Psicología Y Salud, 14(1), 121-134.

Morente, R., Guiu, F., Castells, R., \& Escoda, P. (2017). ANALYSIS OF THE RELATIONSHIP BETWEEN EMOTIONAL COMPETENCES, SELF-.

Nazarzadeh, M., Sarokhani, M., \& Sayehmiri, K. (2014). The Relationship Between Religious Attitudes, Fear of Death and Dying with General Health Condition: A Survey in College Students. Journal of Religion and Health, 54(5), 1672-1680. https://doi.org/10.1007/s10943-014-9903-6 
Ogle, C. M., Rubin, D. C., \& Siegler, I. C. (2014). Events Over the Life Course, 1(4), 426434. https://doi.org/10.1177/2167702613485076.The

Ozbas, A. A., Gurhan, N., \& Kocak, M. (2013). The relation of traumatic childhood experiences with psychological symptoms and self-esteem in physical education and sport students, 79-85.

Páramo, P. (2008). La construcción psicosocial de la identidad y del self. Revista Latinoamericana de Psicologia, 40(3), 539 - 550. Retrieved from http:/ / www.redalyc.org/pdf/805/80511493010.pdf

Pavez Soto, I. (2012). Sociología de la Infancia : las niñas y los niños como actores sociales. Revista de Sociología, 27(27), 81-102. https://doi.org/10.5354/0719-529X.2012.27479

Piedmont, R. L. (1999). Strategies for using the five-factor model of personality in religious research. Journal of Psychology and Theology, 27(4), 338-350.

Piedmont, R. L., Sherman, M. F., \& Sherman, N. C. (2012). Maladaptively high and low openness: the case for experiential permeability. Journal of Personality, 80(6), 1641-68. https://doi.org/10.1111/j.1467-6494.2012.00777.x

Piedmont, R. L., \& Wilkins, T. A. (2013). Spirituality, religiousness, and personality: Theoretical foundations and empirical applications. APA Handbook of Psychology, Religion, and Spirituality (Vol 1): Context, Theory, and Research., 1, 173-186. https://doi.org/10.1037/14045-009

Pierce, J. W., \& Wardle, J. (1997). Cause and effect beliefs and self-esteem of overweight children. Journal of Child Psychology and Psychiatry and Allied Disciplines, 38(6), 645650. https://doi.org/10.1111/j.1469-7610.1997.tb01691.x

Rodriguez, C. M. (2016). Predicting Parent-Child Aggression Risk. Journal of Interpersonal Violence, 88626051662938. https://doi.org/10.1177/0886260516629386

Romero-Martinez, A., Figueiredo, B., \& Moya-Albiol, L. (2014). Childhood history of abuse and child abuse potential: The role of parent's gender and timing of childhood abuse. Child Abuse and Neglect, 38(3), 510-516.

https://doi.org/10.1016/j.chiabu.2013.09.010

Rosés Gómez, M. del R., Raich, R. M. ., \& Mora Giral, M. (2015). Desarrollo y evaluación de la eficacia de dos programas preventivos en comportamientos no saludables respecto al peso y la alimentación. Estudio piloto, 1-177. Retrieved from http://ddd.uab.cat/record/ 128989

Sánchez, D; López, G; Fauquet, J. (2014). Ideal De Belleza Femenino En Los Medios De Comunicación Y Alteraciones De La Conducta Alimentaria. Influencia Y Posibles Soluciones, 437-449. Retrieved from https://idus.us.es/xmlui/handle/11441/36754

Sánchez, N., \& Cuenya, L. (2011). Estudio sobre Maltrato Infantil en Niños y Adolescentes de la Provincia de Buenos Aires. Revista Argentina de Ciencias Del Comportamiento, 3(3), 8-15. Retrieved from http://www.psyche.unc.edu.ar/racc/index.php/comportamiento/article/viewArticle/ 10 $9 \backslash$ nhttps:// revistas.unc.edu.ar/index.php/racc/article/view/5200/5365

Santacruz, G. (2013). El trabajo infantil en Paraguay. Población Y Desarrollo, 72-81.

Schmitt, D. P., \& Allik, J. (2005). Simultaneous Administration of the Rosenberg SelfEsteem Scale in 53 Nations: Exploring the Universal and Culture-Specific Features of Global Self-Esteem. Journal of Personality and Social Psychology, 89(4), 623-642. https://doi.org/10.1037/0022-3514.89.4.623

Scott, E. J., \& Dale, J. (2016). Childhood bullying: Implications for general practice. British Journal of General Practice, 66(651), 504-505. https://doi.org/10.3399/bjgp16X687145 
SELF ESTEEM AND HEALTH-RISK BEHAVIORS AMONG TURKISH LATE ADOLESCENTS Ayçenur Büyukgöze Kavas. (2009), 44(173).

Serrano Muñoz, A., Mérida Serrano, R., \& Tabernero Urbieta, C. (2015). Estudio de la autoestima infantil en función del sexo. Revista Sobre La Infancia Y La Adolescencia, (9), 98-115. https://doi.org/10.4995/reinad.2015.4120

Silverthorn, N., DuBois, D. L., Lewis, K. M., Reed, A., Bavarian, N., Day, J., ... Flay, B. R. (2017). Effects of a school-based social-emotional and character development program on self-esteem levels and processes: A cluster-randomized controlled trial. SAGE Open, 7(3). https://doi.org/10.1177/2158244017713238

Simkin, H., Azzollini, S. \& Voloschin, C. (2014). Autoestima y problemáticas psicosociales en la infancia, adolescencia y juventud. Revista de Investigación En Psicología Social, 1(1), 59-96.

Simkin, H. (2017). Adaptación y Validación al Español de la Escala de Evaluación de Espiritualidad y Sentimientos Religiosos (ASPIRES): la trascendencia espiritual en el modelo de los cinco factores. Universitas Psychologica, 16(2).

Simkin, H., \& Azzollini, S. (2015). Personalidad, Autoestima, Espiritualidad y Religiosidad desde el Modelo y la Teoría de los Cinco Factores. PSIENCIA. Revista Latinoamericana de Ciencia Psicológica, 7(2), 339-361. https://doi.org/10.5872/psiencia/7.2.

Simkin, H., Azzollini, S., \& Voloschin, C. (2014). Autoestima y Problemáticas Psicosociales en la Infancia, Adolescencia y Juventud. \{PSOCIAL\}, 1(1), 59-96.

Simkin, H., \& Etchevers, M. (2014). Religiosidad, espiritualidad y salud mental en el marco del Modelo de los Cinco Factores de la Personalidad. Acta Psiquiátrica Y Psicológica de América Latina, 60(4), 265-275.

Sowislo, J. F., \& Orth, U. (2013). Does low self-esteem predict depression and anxiety? A meta-analysis of longitudinal studies. Psychological Bulletin, 139(1), 213-240. https://doi.org/10.1037/a0028931

Spinhoven, P., Penninx, B. W., Hickendorff, M., Hemert, A. M. Van, \& Bernstein, D. P. (2014). Childhood Trauma Questionnaire : Factor Structure , Measurement Invariance , and Validity Across Emotional Disorders, 26(3), 717-729.

Stein, J. A., Leslie, M. B., \& Nyamathi, A. (2002). Relative contributions of parent substance use and childhood maltreatment to chronic homelessness, depression, and substance abuse problems among homeless women: Mediating roles of self-esteem and abuse in adulthood. Child Abuse and Neglect, 26(10), 1011-1027. https://doi.org/10.1016/S0145-2134(02)00382-4

Ventura, C. A. A., de Souza, J., Hayashida, M., \& Ferreira, P. S. (2015). Risk factors for involvement with illegal drugs: opinion of family members or significant others. Journal of Substance Use, 20(2), 136-142. https:// doi.org/10.3109/14659891.2013.875077

Vlachou, M., Andreou, E., Botsoglou, K., \& Didaskalou, E. (2011). Bully/Victim Problems Among Preschool Children: A Review of Current Research Evidence. Educational Psychology Review, 23(3), 329-358. https://doi.org/10.1007/s10648-011-9153-z

Voloschin, C., Becerra, G., \& Simkin, H. (2016). Bullying escolar, dominancia y autoestima. Una mirada desde la psicología social. Revista de Ciencias Sociales, 92, 62-67.

Zuffianò, A., Alessandri, G., Gerbino, M., Luengo Kanacri, B. P., Di Giunta, L., Milioni, M., \& Caprara, G. V. (2013). Academic achievement: The unique contribution of self-efficacy beliefs in self-regulated learning beyond intelligence, personality traits, and self-esteem. Learning and Individual Differences, 23(1), 158-162. https://doi.org/10.1016/j.lindif.2012.07.010 